choriod plexuses of kitten and chick embryos were obtained on the same culture pattern. These endothelial cells were closely resembling structure (formation and organization) in above each organs. Perfusing the methylen blue and trypan blue solutions, endothelial cells are shown the phagocytic activity earlier than the astrocytes. Under these conditions, all cells were dyed.

In comparing the effects of vasoconstrictors, $\mathrm{Na}-\mathrm{K}$ ion, $\mathrm{Ca}-\mathrm{Mg}$ ion and hypertonic solution on astrocyte and endothelial cell, four points of difference are encountered.

First, serotonin at $50 \gamma / \mathrm{ml}$ produced promoting contraction on endothelial cells and continued this condition after retreatment of normal nutrient solution but no effect on astrocytes. Second, epirenamine hydrochloride at $50 \gamma / \mathrm{ml}$ exhibited rapid contraction of endothelial cells without vasocontraction. After one hour during treatment, endothelial cells expanded flatly and capillaries contracted continuously. But astrocytes were shown reversible contractile reaction. Third, high concentration of Na-ion exhibited cell shrinkage on both cells. However, the reaction to this ion are delayed on the astrocyte and seemed to be asynchronous between both cells, so that the perivascular empty space appeared. Fourth, endothelial cells proved to be more sensitive to hyper-Ca-ion than astrocytes. On the contrary, astrocytes showed irreversible injury to the hypertonic solution (20\% glucose Gey' solution), but reversible on endothelial cells.

From above results obtained in vitro it was established that the cell reaction in perivascular elements, such as Blood-Brain Barrier function, were found to show a marked different points on each by the ions, and also to be proved the responsibility by hypertonic solution.

\title{
23. The Effect of Hypercapnea on Blood-Brain Barrier and Phosphorus Metabolism of the Brain
}

\author{
Yoshihiro Ito, Shonosuke Morita, Yoshiyasu KobaYashI, \\ Yashima Togashi, Yuji Saito, Keiichiro MiURa \\ and Takashi KaTo \\ Department of Neuropsychiatry, Hokkaido University \\ School of Medicine
}

The effect of carbon dioxide inhalation upon blood-brain barrier and phosphorus metabolism has been investigated by macroscopic determination of trypan blue penetration into the brain tissue and by radioautographic determination of radioactive phosphorus $\left({ }^{32} \mathrm{P}\right)$ uptake by the brain tissue. These tracers were injected into the peritoneal cavity of albino rats, and the determination were made 
after the animals were exposed to the air contatining $20 \%$ carbon dioxide fo period of 1, 2 and 5 hours, as well as in control group except $\mathrm{CO}_{2}$ inhalation.

Results obtained from the present study is summarized as follows;

1. The trypan blue penetration into the brain tissue was markedly increasec in all one, two and five hour exposure groups.

2. Marked increase of ${ }^{32} \mathrm{P}$ uptake by the central nervous tissue was noted only in the one hour exposure group. The uptake was noted to be approximately the same as in control group when the animals were exposed to $20 \% \mathrm{CO}_{2}$ for 2 hours duration. Diminution of the uptake was noted in the five hour exposure group.

3. Although the ${ }^{32} \mathrm{P}$ uptake was highest in the paraventricular regions and in the hypothalamic areas in all the groups as reported by other investigators, the rate of increase of the ${ }^{32} \mathrm{P}$ uptake in one hour exposure group was not highest in these areas. The ratio of regional ${ }^{32} \mathrm{P}$ uptake between one hour exposure group and control group was noted to be highest in the cerebellar cortex and white matter, cerebral cortex, and in the basal ganglia.

\title{
24. The Electron Microscopic Studies on the Brain Cortex during Infusion of Hypotonic- and Hypertonic Glucose Solution, Especially in Relation to the Blood-Brain Barrier
}

\author{
Junichi Wakizaka, Shinken Kuramoto, and Toshikazu Fukuda \\ Department of Surgery, Kurume University School of Medicine \\ (Director: Prof. J. Wakizaka, M.D.)
}

The fine structures of the brain cortex of the cats were observed by injection of hypotonic and hypertonic glucose solution to the carotid artery. The capillary vessels of normal brain cortex were almost round, and were surrounded with the processes of astrocytes in the rate of $85 \%$ and others with the other glia cells. Socalled pericapillary space could not be observed.

The group injected with hypotonic solution:

The processes of astroglial cells swollen and the electron density of the cytoplasm was generally low. The findings of capillary vessels indicated irregularly forms pressed by the circumference, and the electron density of capillary lumen was high.

The group injected with hypertonic glucose solution:

The cytoplasm of capillary endothelium was occasionally protruded into the vessel canals, resulting the canal being narrow. Therefore it seems cytoplasmic edema of the endothelial cells. On the other hand, the processes of astroglial cells 